\title{
Erratum to: Initial Approach to Childhood Obesity in Spain. A Multisociety Expert Panel Assessment
}

\author{
Ramon Vilallonga ${ }^{1,2,3}$ • José Manuel Moreno Villares ${ }^{4}$ Diego Yeste Fernández ${ }^{4}$. \\ Raquel Sánchez Santos ${ }^{5}$ - Felipe Casanueva Freijo ${ }^{6}$ - Francisco Santolaya Ochando ${ }^{7}$. \\ Nuria Leal Hernando ${ }^{8}$ • Albert Lecube Torelló ${ }^{9} \cdot$ Luis Antonio Castaño González ${ }^{10}$. \\ Albert Feliu ${ }^{9}$ - Gontrand Lopez-Nava ${ }^{11}$ • Dolores Frutos ${ }^{1}$. Felipe de la Cruz Vigo ${ }^{1}$. \\ Antonio J. Torres Garcia ${ }^{1}$ - Juan Carlos de Ruiz de Adana ${ }^{1}$
}

\section{OBES SURG}

\section{DOI 10.1007/s11695-016-2413-8}

In the original article, an author was missing from the author's list. Dr. Felipe de la Cruz should be added as a co-author for his work in the study design and review process.

The online version of the original article can be found at http://dx.doi. org/10.1007/s11695-016-2413-8

Ramon Vilallonga

vilallongapuy@hotmail.com

SECO Sociedad Española de Cirugía de la Obesidad, Madrid, Spain

2 General Surgery Department, Endocrine, Metabolic and Bariatric Unit, Robotic Surgery, Universitat Autònoma de Barcelona, Universitary Hospital Vall d'Hebron, Passeig de la Vall d'Hebron 119-129, 08035 Barcelona, Spain

3 Spanish Society for Obesity Surgery. General Secretary, Madrid, Spain

4 AEP Asociación Española de Pediatría, Madrid, Spain
5 AEC Asociación Española de Cirugía-Sección Obesidad, Madrid, Spain

6 SEEDO Sociedad Española para el Estudio de la Obesidad, Madrid, Spain

7 Consejo General de Colegios Oficiales de Psicólogos, Madrid, Spain

8 SECP Sociedad Española de Cirugía Pediátrica, Madrid, Spain

9 SEEN Sociedad Española de Endocrinología y Nutrición, Madrid, Spain

10 SEEP Sociedad Española de Endocrinología Pediátrica, Madrid, Spain

11 SEPD Sociedad Española de Patología Digestiva, Madrid, Spain 